\title{
Une classe de guerre à Vinassan, Aude - juin-juillet
} 1918

\section{Rémy Pech}

\section{OpenEdition}

1 Journals

\section{Édition électronique}

URL : http://journals.openedition.org/trema/1894

DOI : 10.4000/trema.1894

ISSN : 2107-0997

\section{Éditeur}

Faculté d'Éducation de l'université de Montpellier

\section{Édition imprimée}

Date de publication : 1 décembre 1997

Pagination : 155-164

ISSN : 1167-315X

\section{Référence électronique}

Rémy Pech, « Une classe de guerre à Vinassan, Aude - juin-juillet 1918 », Tréma [En ligne], 12-13| 1997, mis en ligne le 24 novembre 2010, consulté le 21 avril 2019. URL : http://journals.openedition.org/ trema/1894; DOI : 10.4000/trema.1894

Ce document a été généré automatiquement le 21 avril 2019.

Trema 


\title{
Une classe de guerre à Vinassan, Aude - juin-juillet 1918
}

\author{
Rémy Pech
}

1 Vinassan, humble bourgade de la plaine narbonnaise, située à l'orée du petit massif de la Clape, avait 717 habitants en 1911. Sa population fléchit à 704 habitants en 1921 après le déroulement du grand cataclysme. 27 jeunes du village, soit $3,74 \%$, chiffre proche des estimations faites au plan national et presque identique au 3,77\% départemental, ne sont pas revenus du front. Dans le même temps, les premiers «Espagnols » - tous catalans ou valenciens - se sont installés, mais aucun enfant de ces nouveaux venus n'apparaît dans le document étudié.

2 L'instituteur Charles Tort approche, en 1918, de la fin de sa carrière. Celle-ci s'est déroulée pour l'essentiel à Vinassan (nommé en 1882, il s'y marie en 1885 et y exerce jusqu'en 1924). En 1918, il a 59 ans et son fils Alphonse, instituteur public comme lui, est au front.

3 Le « cahier de roulement » de sa classe est un document étonnant. Le titre imprimé sur la couverture révèle évidemment une utilisation officielle et universelle. Tenu par chaque élève à tour de rôle, il a sans doute été institué pour que l'Inspecteur primaire, au cours de sa visite, puisse prendre, à sa lecture, la mesure du véritable niveau de la classe sans se laisser impressionner par les prestations orales des élèves les plus brillants, inévitablement " chauffés » pour cette circonstance par leur maître. Mais au-delà de ce contrôle, le cahier de roulement me paraît exprimer l'identité de la classe: c'est le témoignage du travail collectif, le document où chaque petit écolier a mis tout son cœur. Un peu, il est vrai, pour se mettre lui-même à son avantage, mais surtout pour faire honneur à sa classe, à ses parents, à son village.

Le cahier couvre la période du mardi 11 juin au mercredi 24 juillet 1918, soit 33 jours de classe. Il se compose de 30 feuillets rédigés recto-verso par une dizaine d'élèves de la première et deuxième division du cours moyen, qui suivaient, semble-t-il, les mêmes leçons. Le nombre des scripteurs - sept sont identifiés et deux ou trois autres, non identifiés, sont probables - n'a pu être déterminé avec certitude. Nous ignorons si le tour 
de rôle établi était l'objet d'une sélection délibérée par le maitre. La présence, à 5 reprises, du « bon élève » Roger Fouchac (classé en première division à l'âge de dix ans), futur cadre de banque, celle de mes deux oncles René et Gabriel Fabre, le premier futur secrétaire de mairie, le deuxième viticulteur avisé amateur d'opéra et de littérature, m'inclineraient à détecter un certain souci de la qualité. Quelques prestations sont d'une grande médiocrité, mais il est vrai que l'instituteur pouvait être valorisé aussi par la mise en évidence de l'hétérogénéité culturelle des enfants qui lui étaient confiés.

Charles est alors un instituteur chevronné. A 59 ans, il est le premier chaînon d'une dynastie de quatre générations d'enseignants. Arpenteur et secrétaire de mairie, c'est aussi un viticulteur attentif, qui prêche d'exemple en soignant les vignes de sa femme, héritière d'un petit propriétaire de Vinassan. Aimé, mais assez redouté de ses élèves dont l'un au moins vit encore, il était surnommé familièrement " pelhot "'allusion à une phrase qu'il aurait répété rituellement en fin de journée en incitant un élève à lui remettre un chiffon pour effacer le tableau. Au passage, ceci témoigne sans doute de l'utilisation de l'occitan, peut-être pas dans le cours de la classe mais pour enclencher - de manière résignée ou simplement consciente, on ne peut aujourd'hui que le supposer - le retour évident à la lenga maire après une journée d'immersion obligatoire dans la langue française.

Dans son humilité, le cahier de roulement de Vinassan intéresse l'historien à plus d'un titre. Je n'ai pas eu le temps, et sans doute ma compétence serait-elle problématique, d'évaluer l'impact de la guerre sur la production des occitanismes, les modifications de vocabulaire ou de syntaxe induites par le grand chamboulement. Ceci pourra être esquissé plus tard si je retrouve d'autres cahiers « de guerre », présentement égarés. Mais je crois possible et nécessaire de tenter, à l'instar d'autres chercheurs ${ }^{2}$, une analyse de contenu qui puisse témoigner à la fois de la résistance à l'événement d'un système pédagogique éprouvé et du degré d'instrumentalisation de ce système par une autorité politique toute entière tendue vers l'accomplissement d'une guerre entrée dans sa phase décisive. En réponse à ces deux démarches, peuvent se révéler les réactions des enfants aux sollicitations qui leur sont faites dans un cadre certes encore familier et sécurisant, mais néanmoins sensible aux angoisses et aux fracas de la grande tuerie.

\section{Les permanences}

\section{Un programme fidèlement suivi}

7 L'ordre bien établi de la pédagogie primaire ne semble pas, à la fin de l'année scolaire 1917-18, avoir subi de modification sensible. Ce diagnostic peut être formulé grâce aux comparaisons avec un lot de cahier du même niveau, résultant pour 1899-1900 de l'enseignement du même instituteur dans le même village.

8 La première leçonest essentiellement constituée par une courte intervention du maître, un échange avec un élève, l'inscription d'une maxime au tableau. Ces trois temps sont appliqués à deux disciplines complémentaires, savamment alternées :

- lundi, mercredi et samedi : la morale.

- mardi et vendredi : l'éducation civique.Cet intitulé est ici substitué à celui d'« instruction civique ", en vigueur dans les cahiers des années 1900. L'éducation civique occupe donc le début de deux journées scolaires sur cinq. 
Le lundi 8 juillet, le mardi 9 et le samedi 20 juillet (soit 3 jours sur 33, moins de $10 \%$ ) Charles Tort a substitué à cette introduction de la journée une leçon de guerre aux thèmes d'ailleurs assez peu belliqueux quoique mobilisateurs :

- Il faut produire

- L'effort maritime

- Les Alsaciens-lorrains sont Français.

10 Nous ne pouvons aller très loin dans l'analyse du contenu des leçons de morale, qui ne font pas l'objet de notes, à moins qu'elles ne soient reproduites dans un cahier spécial non conservé.

11 C'est ensuite la grammaire, qui consiste pour l'essentiel à étudier les verbes et leur conjugaison, quelques séances étant consacrées aux conjonctions et prépositions, mais c'est la fin de l'année et bien d'autres subtilités de notre langue avaient dû être traitées auparavant. J'ai observé une liaison certaine avec la leçon précédente, comme si les exemples choisis avaient pour fonction, en plus d'exercer l'enfant à la bonne pratique de la langue nationale, d'illustrer les valeurs inculquées quelques instants avant. La leçon de grammaire théorique, incluant sans doute quelques interrogations "au tableau», est invariablement suivie d'une dictée, celle-ci embrayant sur une analyse logique ou une composition française ou rédaction originale.

12 L'après-midi est systématiquement consacré au calcul. Charles Tort est particulièrement attentif à poser des problèmes utiles, imagés comme nous le verrons.

13 La fin de journée est consacrée aux autres matières. Des lectures utilisent généralement des fables, des récits de voyage ou des évocations pittoresques, mais pouvant devenir patriotiques comme Les villages alsaciens (fol. 10).

14 L'histoire et la géographie(huit séances d'histoire et trois de géographie), laissent parfois place aux sciences(deux séances, plus une d'agriculture). Ces leçons représentent un moment important puisque, préparant le retour à la maison elles sont susceptibles d'« exportation » dans le milieu familial.

\section{Un contenu utilisant au maximum le milieu}

15 L'analyse du cahier de Vinassan me paraît conforter les remarques de J.-F. Chanet concernant «la formation adaptée au milieu local $»^{3}$. Je m'en tiendrai ici à quelques exemples.

La majorité des problèmes d'arithmétique et de géométrie puisent leurs thèmes dans des exemples locaux. Il s'agit de mesurer un jardin, de l'acheter ( $f \circ l .1$ ). De même pour un champ (fol. 7) et une vigne, dont la description est en tous points conforme aux lopins alors cultivés par les vignerons du Narbonnais (fol. 15) et dont la procédure d'acquisition est également tout à fait vraisemblable :

«Mon père achète une vigne rectangulaire de $80 \mathrm{~m}$ de long sur $50 \mathrm{~m}$ de large au prix de $75 \mathrm{f}$

l'are. Il paie comptant la moitié du prix et l'autre moitié 8 mois après avec l'intérêt à $5 \%$.

Combien paie-t-il la $2^{e}$ fois ? " ${ }^{4}$

17 Le calcul du temps employé en fonction de la vitesse de déplacement et de la distance parcourue met en scène un enfant se rendant à pied à Narbonne, distante de $6 \mathrm{Km} 780$ (fol. 19). Cette performance a été accomplie maintes et maintes fois par tout Vinassanais né avant 1950. Les quantités à évaluer, avec calcul de frais et de bénéfice, sont évidemment fondées sur le négoce du vin : 
"Un restaurateur reçoit 18 pièces de vin contenant chacune 225 litres. ${ }^{5}$ Chaque pièce coûte $89 f$ d'achat, $6 f 35$ de port, $16 f 60$ d'entrée, $3 f 50$ de commission, of 50 d'encavement. S'il vend le litre of 80 , quel bénéfice fera-t-il ? ${ }^{6}(f \circ l .5)$

18 Le bénéfice ainsi évalué représente $1152 \mathrm{f} 90$ pour un déboursé de $2087 \mathrm{f} 10$, opération tout à fait lucrative, mais qui ne pouvait choquer un enfant habitué aux fréquentes allées et venues des courtiers, commissionnaires, négociants chez ses parents ou voisins.

19 Plus amusant est le problème évoquant la fabrication d'une mixture assez suspecte :

«Peu de temps avant la moisson (le scripteur, Gabriel Fabre, avait dû raturer le très significatif boisson primitivement écrit) un fermier a fait mélanger 150 litres de vin à of 50 le litre et 275 litres d'un autre vin à $0 f 40$ le litre et 100 litres d'eau. Il a payé $4 \mathrm{f}$ au journalier chargé de ce travail. A combien lui revient le litre de cette boisson destinée à sa famille?

Réponse : of $36 . »($ fol. 28) rurales, relever deux dictées concernant les céréales. La dictée intitulée Avant la moisson est extraite de La terrede Zola et évoque : "La Beauce» (qui) « déroule ses blés mûrs sous un ciel de flamme.

C'était une mer blonde incendiée, qui semblait refléter le flamboiement de l'air, une mer roulant sa houle de feu, au moindre souffle. Rien que du blé, sans qu'on aperçût une maison ni un arbre, l'infini du blé: parfois, dans la chaleur un calme de plomb endormait les épis, une odeur de fécondité fumait et s'exhalait de la terre. ». (fol. 19) ${ }^{8}$ sur la p. 319 du manuel, porte sur Le battage du blé en Provence (fol. 30).

Calligraphiée par le bon élève Roger Fouchac, mais tout de même émaillée de 6 fautes d'orthographe, la dictée Figues fraîches et figues sèches,peut-être du cru de Charles luimême, constitue tout à la fois une recette pratique dans un village ou le "grappillage » 
des figues représentait une activité saisonnière bien instituée pour les familles pauvres, une « leçon de choses » et une mesure du particularisme méditerranéen.

"Les figues sont mûres lorsque le suc âcre et laiteux qu'elles contiennent est changé en une eau limpide et sucrée. Elles deviennent alors molles, charnues et pendantes. Dans le Midi, celles qui sont destinées à être mangées fraîches sont cueillies un peu avant leur maturité; sous le climat parisien, elles ne sont jamais trop mûres [...]. » (fol. 24)

L'essentiel - en quantité - des leçons de calcul et des dictées met en œuvre une matière vivante antérieure à la guerre ou en tous cas ignorant celle-ci. Il ne semble pas non plus que la très importante leçon de morale inaugurant la journée témoigne d'une obsession de la guerre. Nous n'avons pas, c'est vrai, de notes détaillées rapportant les mots proférés par le maître et plusieurs thèmes se prêtent à une exploitation patriotique. Nous reproduisons ci-après la liste intégrale des dix leçons de morale de cette fin d'année scolaire :

- «Soyez patients. Soyez obstinés

- L'effort paysan

- Respect des biens d'autrui

- Respect de la pensée. Des opinions

- La tolérance

- La politesse

- La charité

- La bonté

- Les nationalités opprimées (1 leçon prolongée le surlendemain au titre de l'éducation civique)

- L'aumône

- Le dévouement. Le sacrifice

- Fraternité. Solidarité. Philanthropie "

A contrario, l'insertion, entre charité et bonté, mais toujours sous la rubrique Morale, de la leçon sur "Les nationalités opprimées " (fol. 20-21), est évidemment dictée par l'actualité, la lutte pour les droits des nations, mise en exergue par les fameux «14 Points » de Wilson (janvier 1918), venant englober et renforcer la revendication inlassablement ressassée depuis 1871 de l'Alsace et de la Lorraine. L'intitulé, le 24 juillet, de l'ultime leçon Fraternité. Solidarité. Philanthropie (fol. 30), est un message porteur d'espoir au moment précis où la guerre se déchaine à nouveau en Champagne et en Ile de France. Nous observons en cette fin d'année une véritable fusion des leçons de morale et d'éducation civique, qui ne paraît pas seulement dictée par la nécessité d'épuiser le programme. Il s'agit, sans doute davantage, d'arrimer le message de la mobilisation à l'inculcation quotidienne des préceptes moraux.

\section{Les changements : Un enseignement tendu vers la guerre}

30 La guerre proprement dite n'intervient qu'exceptionnellement. On peut en trouver la trace dans cette lecture « du samedi » (spécialement destinée à la diffusion aux adultes ?) sur "Les assassins de la mer» (fol. 13) que l'on peut supposer consacrée aux affres de la guerre sous-marine plutôt qu'à des requins ou à quelque danger pour l'écologie, alors très ignorée. Plus fréquemment, nous avons relevé l'occurrence de thèmes insérant le drame commun dans l'actualité du village. Ainsi, une Composition française évoquant une visite à un frère blessé (fol. 2 et 3). 
"Vos parents sont partis auprès de votre frère aîné blessé sur le front et évacué à l'hôpital et ils vous ont fait des recommandations que vous avez promis de suivre.

«Racontez leur départ précipité. Dites quelles recommandations ils vous ont faites et dites ce que vous avez fait pendant leur absence."

31 On ne saurait mieux préparer les enfants à une éventualité hélas alors trop vraisemblable. Le jour suivant, c'est la dictée Têtes blondes et têtes blanches, où l'on peut lire, sans que la nostalgie de ce texte manifestement «d'avant-guerre » estompe la nécessaire solidarité des générations cruellement mise à l'ordre du jour par la guerre :

"Les têtes blondes des petits enfants et les têtes blanches des vieillards se recherchent et se comprennent naturellement. Pendant que l'adolescent court à ses travaux ou à ses plaisirs, les vieillards sont à l'écart avec leurs souvenirs et les enfants avec leurs jeux. L'été, lorsque le foin sèche ou que la moisson appelle tous les bras valides, les champs fourmillent de travailleurs, mais les villages sont vides. "Il n'y reste que les vieux et les petits. Au pas des portes, sur des bancs de bois, se tiennent de vieilles femmes, à la tête branlante, des grands pères, le menton appuyé sur leur bâton, regardent autour d'eux s'agiter des groupes d'enfants.

"Les villages sont vides... Il ne reste que les vieux et les petits. » (fol. 3) ${ }^{9}$

Mais c'est la dictée Patriotisme, aux accents forts martiaux, voire chauvins (fol. 7 -journée du 18 juin située peu après le déchaînement de l'offensive allemande sur la Marne), qui nous paraît la plus significative :

"Suivant la loi d'amour aimons tous les hommes mais ayons une préférence pour nos compatriotes. Nous appartenons à la même race, les traits de notre physionomie, notre tournure d'esprit, nos qualités morales et aussi nos défauts décèlent une commune origine. Nous descendons des Francs \& que la mort abat et non la crainte, chez qui le courage vit encore quand le souffle vital s'est éteint >... Nous devons aux Romains notre langue, nos mœurs, nos institutions, mais c'est sans doute du sang gaulois généreux et vaillant qui coule dans nos veines $»^{10}$.

Même si aucune allusion n'est faite à l'arrivée des troupes du général Pershing, le sujet de la rédaction du 3 juillet, venant après une leçon de morale consacrée à la Commémoration de l'Indépendance américaine, n'est pas détachée du contexte :

"Pourquoi demain tous les édifices publics seront-ils pavoisés. De quelles façon vous associerez-vous à cette commémoration?" (fol. 18).

Quelques jours après est mentionnée (fol. 21) une lecture En Louisiane,qui a probablement été le support d'une nouvelle célébration de l'amitié, et même cette fois de la parenté entre les deux peuples engagés dans la guerre.

Les thèmes d'éducation civique sont plus éclairants encore car ils insèrent la réalité de la guerre dans l'enseignement traditionnel de la République. En voici la liste :

- Laboratoire. Usine. Guerre.

- Organisation actuelle de la force publique

- Organisation des armées de terre

- Armée

- Armée de mer

- Rapports internationaux

- Etat actuel des rapports internationaux

- Rapports internationaux. L'avenir.

Ce dernier thème est articulé sur un exercice de grammaire qui met en perspective la paix prochaine. Malheureusement la rédaction en est à peine lisible tant la décomposition du texte en sujets, verbes et compléments, but de l'exercice, est défectueuse. L'Allemagne est encore qualifiée de brutale et envieuse, mais la foi en l'avenir se manifeste par la phrase : 
"Un courant de progrès amènera tôt ou tard la paix dans le monde » et par une conclusion elle aussi très " wilsonienne » : " chaque peuple d'Europe pourra panser ses plaies " (fol. 27).

Situé le 23 juillet, presque en conclusion du cahier - et de l'année - cet exercice vient compléter le texte suivant et les développements qui lui sont annexés, proposés aux élèves quelques semaines plus tôt, le 28 juin (fol. 15-16) ${ }^{11}$.

"Ce que défendent nos soldats.

"Ce que vous défendez c'est notre patrimoine moral, nos moeurs, nos usages, nos lois, nos coutumes, nos croyances, notre tradition, ce sont les ceuvres de nos sculpteurs, de nos architectes, de nos peintres, de nos graveurs, de nos orfèvres, de nos émailleurs, de nos verriers, de nos tisserands, ce sont les chants de nos musiciens, c'est le parler maternel qui durant huit siècles, avec une ineffable douceur, coula sans tarir des lèvres de nos poètes, de nos orateurs, de nos historiens, de nos philosophes. Ce que vous défendez, c'est le génie français qui éclaira le monde et porta la liberté aux peuples et c'est cet esprit généreux qui fait tomber les Bastilles.»

Anatole France.

Ce texte du grand écrivain républicain apostrophant les héros du front est suivi de questions et de réponses éloquentes dans leur naïveté :

«1 - Pourquoi l'Allemand se bat-il et pourquoi nous battons-nous?

"2-Qu'est-ce que le patrimoine? Les mots de la même famille."

Et voici les réponses :

"1-Parce que les Allemands (barré: n'avaient pas assez avec l'Alsace et la Lorraine) se battent pour leur empereur et n'avaient pas assez d'argent pour leur nourriture, alors l'Allemagne nous a déclaré la guerre. Nous nous battons parce que nous voulons pas que la France devienne allemande."

On ne saurait opposer plus simplement la cupidité germanique (fondée sur une idée de précarité économique si peu conforme à la réalité) et l'esprit défensif de la mobilisation française.

«2-C'est du bien du père et de la mère.

Patrie, Patriote, Patriotique, Patriotisme, Patrologie."

Nous avons peu à dire sur les leçons d'histoire, sinon pour signaler le retard dans le traitement du programme, probablement provoqué par des leçons de guerre ou des mises au point d'actualité. C'est Louis XV qui vient ponctuer l'année, en deux leçons. Charles Tort s'est, à l'évidence, trouvé contraint de supprimer la Révolution et l'ensemble du XIX siècle, alors que son cours d'histoire 1899-1900 se terminait par l'actualité la plus récente, soit Casimir-Perier et Félix Faure. Seule entorse, mais si lourde de sens, au déroulement chronologique, la leçon Dernières années de Napoléon III. Guerre franco-allemande, s'intercale entre Louis XIII et Richelieu et La royauté absolue. La cour du roi.Le maître ne peut décemment passer sous silence l'épisode qui constitue la préface et la justification du drame en cours de déroulement.

Passons rapidement sur les questions de géographie, classiquement concentrées en cette fin d'année sur les colonies, avec une insistance particulière sur les colonies d'Afrique. L'étalage de territoires complaisamment énumérés - et laborieusement orthographiés par les petits Languedociens - participe sans doute de la mise en confiance de l'opinion à la fin d'une guerre interminable, à l'issue alors encore très incertaine. Mais c'est aussi plus simplement la mise en scène de l'une des œuvres majeures de la République, dont l'utilité est alors en train de se confirmer à l'épreuve d'une guerre grande consommatrice en hommes. 
Plus significatifs, puisque résultant davantage du choix du maître, les verbes conjugués et étudiés trahissent les préoccupations du moment. Bornons nous à les énumérer par séances successives :

- «savoir, sortir, servir ;

- suffire, suivre, tenir ;

- voir, voler (il s'agit de hannetons et non d'avions!);

- traiter, vaincre, valoir;

- éteindre;

- vêtir, vivre, voir ;

- courir ;

- vêtir, vivre (2 fois);

- prédire, contredire, redire.»

- avoir, être;

- respecter, convenir;

- raisonner, corriger;

- activer, accéder, actionner; accepter;

- dépendre, triompher;

- amener, passer, panser;

- échapper;

- lasser;

Exceptés ces deux derniers verbes (col. de dr.) qui peuvent évoquer, avec les rêves des prisonniers, la fatigue profonde des populations et la séquence prédire, contredire, redire, verbes de doute autant que d'espérance, Charles Tort n'a mis sur les lèvres des enfants de Vinassan que des verbes exprimant la patience, la ténacité et la confiance en la victoire prochaine. On peut toutefois s'interroger sur l'effet de l'étude du mot sang et de ses dérivés, qui complète la dictée Patriotisme déjà évoquée : l'élève a mentionné sanger ${ }^{12}$, sanguin, sanguinaire, saigner, sangsue (fol. 8).

Mentionnons, pour finir les leçons de sciences: «Métaux - Fer. Le fer, le cuivre, le zinc, l'étain.»

Même si l'on tient compte de l'épuisement d'un programme qui devait comporter des éléments de biologie et de chimie, cette information sur les ressources métalliques, tranche sur le «ruralisme » de la plupart des leçons. Elle en dit long sur la mobilisation économique presque désespérée, alors à l'ordre du jour.

\section{Conclusion : une mobilisation tranquille}

Excepté le choix de la dictée sur Le patriotisme, Charles Tort ne semble pas avoir manifesté un zèle nationaliste particulier. Ce que nous savons de lui, par la mémoire qu'il a conservée dans le village, peut se résumer ainsi: il s'agissait d'un bon républicain, collaborateur loyal, en tant que secrétaire de mairie, du maire socialiste élu puis constamment renouvelé à partir de $1900^{13}$. La force du patriotisme qui se dégage de ces pages tient au fait d'avoir été instillé dans le corps même de leçons ordinaires plutôt que déclamé de tonitruante manière. Et c'est peut-être de cette façon que la France et la République ont été défendues, sans que leur marche vers le progrès, toujours ardemment souhaitée, soit vraiment ralentie, et sans que la "petite patrie " soit effacée par le sacrifice alors exigé par la grande, mais au contraire intégrée, dans ses chiffres, ses images et ses valeurs, à la mobilisation suprême. 


\section{NOTES}

1. Occitan : chiffon.

2. Pour une commune peu éloignée de Vinassan, mais pour un temps décalé, lire l'œuvre pionnière de CAZALS R., Les écoliers de Tournissan,Toulouse, Éd. Privat, 1978, 156 p.

3. CHANET J.-F., L'école républicaine et les petites patries,Paris, Éd. Aubier, 1996, 426 p. Lire en particulier le Ch. IV, « une formation adaptée au milieu local ? » p. 145-175.

4. Texte reproduit intégralement.

5. Il s'agit de la barrique, ou bordelaise, alors usuelle pour les livraisons à particuliers.

6. Texte reproduit intégralement.

7. Les chiffres fournis tiennent compte de l'inflation de guerre et des niveaux de salaire engendrées par celle-ci.

8. Texte intégralement reproduit.

9. Idem.

10. Idem.

11. Idem.

12. Verbe francitan pour; changer.

13. Source : témoignages divers, corroborés par les registres de délibérations et l'état-civil de Vinassan.

\section{RÉSUMÉS}

Not available

\section{INDEX}

Mots-clés : Aude, école primaire, enseignement, IIIe république, première guerre mondiale, ruralité

Keywords : first world war, primary school, rural context, rural life, teaching, third republic

\section{AUTEUR}

\section{RÉMY PECH}

Université de Toulouse Le Mirail 